Trauma Berufskrankh 2009 · 11 [Suppl 2]: 184-186

DOI 10.1007/s10039-008-1465-y

Online publiziert: 7. Februar 2009

(c) Springer Medizin Verlag 2009

\author{
F. Wagner \\ BG-Unfallklinik Murnau
}

\title{
Laboruntersuchungen zur Osteitisdiagnostik
}

Cut-off-Wert für einzelne Laborparameter existiert nicht. Im Behandlungsverlauf ist das CRP der entscheidende Parameter, um einen günstigen Heilungsverlauf anzuzeigen.

Generell ist zu klären, in welcher Phase der Entzündungskaskade sich der Patient gerade befindet ( $\mathbf{\bullet}$ Abb. 1, 2, 3):

- Handelt es sich um eine akut aufgetretene Knocheninfektion oder liegt ein jahrzehntelanger chronischer Knocheninfekt vor?

- Handelt es sich um eine regionale Entzündung oder liegen bereits eine systemische Körperantwort in Form einer SIRS („systemic inflammatory response syndrom") oder einer Sepsis oder gar ein septischen Organversagen vor?

Im Folgenden sollen die gebräuchlichen Laboruntersuchungen auf ihre Eignung dargestellt werden [1].
Blutbild (BB)

Die heute automatisierte Blutbildbestimmung wird im EDTA-Blut vorgenommen. Hierbei werden die Menge und Größe von Erythrozyten, die Anzahl der Leukozyten und der Thrombozyten aufgelistet. Unterschiedliche Normwerte ergeben sich bezüglich des Lebensalters und diverser hämatologischer Grunderkrankungen.

Gemeinhin als erhöht gelten Leukozytenzahlen über $10 \times 10^{3}$, und eine Thrombozytenzahl unter $150 \times 10^{3}$ gilt als vermindert. Die Sensitivität des Blutbildes zur Diagnostik einer Osteitis wird mit etwa 90\% angegeben, die Spezifität dafür ist gering. Eine mikrozytäre Anämie kann u. a. Ausdruck einer chronischen Infektanämie sein.

Störgrößen ergeben sich durch Blutungsanämien, hämatologische Grunderkrankungen und die heparininduzierte Thrombopenie (HIT).

Die Cochrane-Analyse erbrachte je nach Sortierung der Suchwörter 128-185 Treffer. Es fand sich keine Arbeit, in der eine Grad-1- oder -2-Evidenz für einzelne Laboruntersuchungen zur Diagnostik der Osteitis angegeben worden war.

Die Pubmed-Analyse zeigte 4-120 Treffer. Lediglich die Arbeiten von Unkilla-Kallio et al. [10, 11] zeigten eine Grad-2Evidenz, dass das CPR (C-reaktives Protein) den Verlauf einer kindlichen hämatogenen Osteomyelitis signifikant besser anzeigt als Blutbild und -senkung.

In den Standardwerken der Unfallchirurgie und rekonstruktiven Extremitätenchirurgie wird zur Primärdiagnostik einer Osteitis auf Blutbild, CRP und Blutsenkungsgeschwindigkeit verwiesen. Ein



Abb. 14 Klinische Zeichen der Entzündung 


\section{Differenzialblutbild (diffBB)}

Es wird ebenfalls automatisiert im EDTA-Blut untersucht: Dabei werden die Leukozyten nach ihrer Morphologie eingeteilt und gezählt. Ein Übermaß an segmentkernigen Leukozyten gibt einen Hinweis auf eine bakterielle Entzündung. Es liegen keine Angaben zur Sensitivität und Spezifität vor.

\section{Blutsenkungsgeschwindigkeit (BSG)}

Sie wird nach der Westergren-Methode durch Sedimentation antikoagulierten Na-Zitrat-Vollblutes allein durch Schwerkraft bestimmt. Als pathologisch gelten Senkungen $>20 \mathrm{~mm} / \mathrm{h}$. Die Methode ist einfach, billig, aber unspezifisch (Sensitivität etwa $60 \%$ ). Als Verlaufskontrolle ist sie auch in der Osteitisbehandlung etabliert, der 2-h-Wert ist ohne Aussagekraft.

\section{C-reaktives Protein (CRP)}

Es wird maschinell im Serum bestimmt. Werte $<0,3 \mathrm{mg} / \mathrm{dl}$ gelten als normal. Das CRP steigt binnen $24 \mathrm{~h}$ nach einer Entzündung an, kann also in einer hoch akuten Phase noch normal sein. Die Produktion ist abhängig von der Leberfunktion, kann also in einer späten Sepsisphase mit Organversagen nicht mehr als Verlaufparameter herangezogen werden. Weitere Störgrößen ergeben sich bei aktiviertem Komplementsystem, Pankreatitis sowie Tumor- und rheumatischen Erkrankungen. Als Verlaufsparameter einer Entzündungsaktivität ist das CRP bestens evaluiert. Es fällt in wenigen Tagen bei Genesung ab und bleibt bei chronischer Infektion erhöht. Es werden eine Sensitivität von $>90 \%$ und eine Entzündungsspezifität von 95\% angegeben. Eine Zuordnung zur Osteitis ist nicht möglich.

\section{Prokalzitonin (PCT)}

Es wird ebenfalls im Serum bestimmt (http://de.wikipedia.org/wiki/procalcitonin). Werte $>0,5 \mathrm{ng} / \mathrm{ml}$ gelten als erhöht. Das PCT ist chemisch die Vorstufe des Kalzitoninhormons und steigt bei schweren Infektionen oder Sepsis binnen Stunden hoch an. Die PCT hilft bei der Differen-

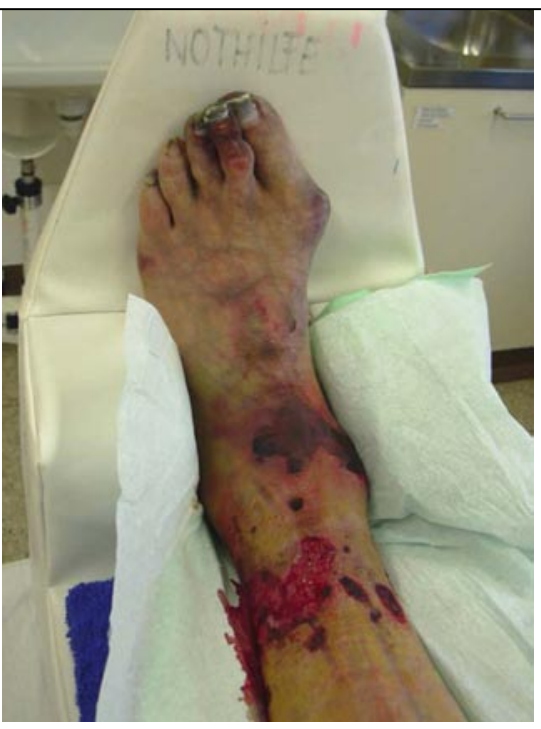

Abb. $2 \Delta$ Akute Osteitis nach operativ versorgter Pilon-tibiale-Fraktur

zierung zwischen bakteriellen und viralen Infektionen und ist ein unabhängiger Prognosefaktor für das Multiorganversagen. Als Screeningtest ist sie ungeeignet, da sie teuer und bezüglich einer Osteitis unspezifisch ist.

\section{Akutphaseproteine}

In der Sepsisforschung etabliert sind Zytokine, PMN-Elastase (polymorphkernige Elastase), Serumamyloid und Antithrombin (AT) III. Keiner dieser aufwändigen Tests ist zur Diagnostik der Osteitis geeignet. Die höchste Spezifität besitzt noch die PNM-Elastase [6], allerdings werden die Sensitivitäten des CRP nicht erreicht.

\section{Diskussion}

In der Unfallklinik Murnau werden als Aufnahmelabor bei Verdacht einer Knochen- und Gelenkinfektion das Blutbild, die Gerinnung, die Nieren- und Leberwerte sowie das CRP bestimmt. Bei Verdacht einer septischen Komplikation werden diffBB und PCT mitbestimmt sowie mehrere Blutkulturen abgenommen [12].

Zur Verlaufskontrolle bei Sanierung einer Osteitis werden Blutbild und CRP bestimmt [8]. Diese Regeln gelten gleichermaßen für Kinder [4].
Trauma Berufskrankh 2009 · 11[Suppl 2]:

184-186

DOI 10.1007/s10039-008-1465-y

(c) Springer Medizin Verlag 2009

\section{F. Wagner}

\section{Laboruntersuchungen zur Osteitisdiagnostik}

\section{Zusammenfassung}

Die Diagnose einer Osteitis wird klinisch gestellt. Laborchemische Untersuchungen unterstützen nur die Diagnosefindung, schließen aber eine Knochen- oder Gelenkentzündung nicht mit Sicherheit aus. Geeignete Blutuntersuchungen sind das Blutbild, das CRP (C-reaktives Protein) und die Blutsenkungsgeschwindigkeit. Der Prokalzitonintest zeigt die septische Komplikation zuverlässig an. Als Verlaufsuntersuchung in der Osteitisbehandlung genügen Bestimmungen von CRP und Blutsenkung.

\section{Schlüsselwörter}

Osteitis - Osteomyelitis - C-reaktives Protein . Leukozytose · Prokalzitonin

\section{Laboratory tests to diagnose osteomyelitis}

\section{Abstract}

The diagnosis of osteitis is made in a clinical setting. While laboratory examinations are able to support the diagnosis, they are not able to reliably rule out bone or joint inflammation. Appropriate blood tests include full blood count, C-reactive protein (CRP) and erythrocyte sedimentation rate. A procalcitonin test will reliably show any septic complications. CRP and erythrocyte sedimentation rates are sufficient to monitor the course of osteomyelitis therapy.

\section{Keywords}

Osteitis · Osteomyelitis · C-reactive protein . White blood cell count . Procalcitonin 


\section{Diagnostik der Osteitis}

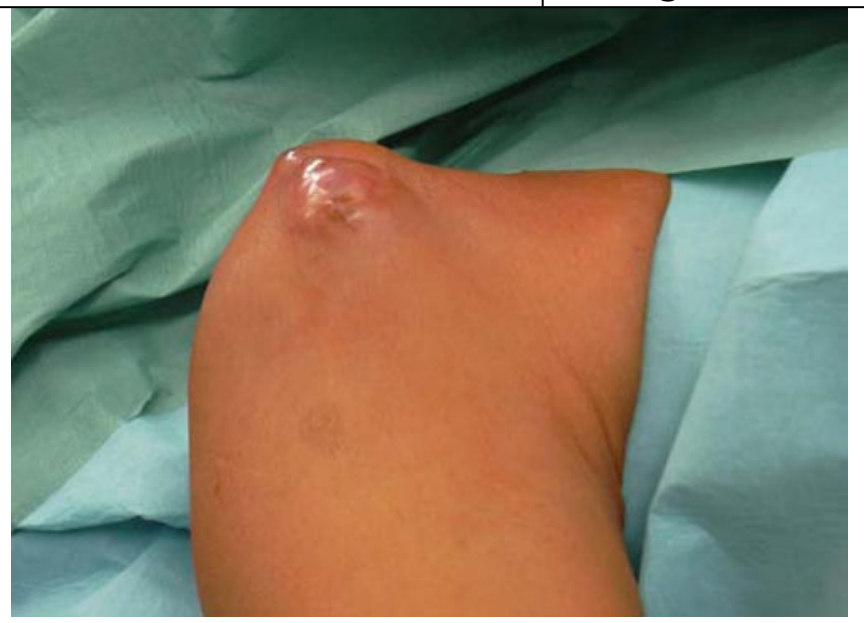

Abb. $3<$ Schulter-

empyem

\section{Fazit für die Praxis}

Zur Diagnosefindung einer akuten Osteitis oder Osteomyelitis braucht es das klinische Bild von Rötung, Schwellung, Schmerz, Überwärmung und Funktionsverlust sowie Röntgen- und nuklearmedizinische Untersuchungen ( $($ Abb. 3). Diffizile Blutuntersuchungen sind nicht notwendig. Eine Knochen- oder Gelenkinfektion führt zu einer lokalen und systemischen Entzündungsreaktion, die sich laborchemisch in einer Erhöhung der Akutphaseproteine und der Leukozytenzahl widerspiegelt. Selten führt diese Entzündung zur Sepsis. Den Beginn einer solchen zeigt die Erhöhung des Prokalzitoninspiegels an. Als Verlaufsparameter bei der Behandlung von Knochenund Gelenkinfektionen haben sich das CRP und die Blutsenkungsgeschwindigkeit bewährt. Bei langen komplizierten Krankheitsverläufen sinkt die Zuverlässigkeit der Blutuntersuchungen weiter ab. Bei chronischen fistelnden Osteitiden können laborchemische Entzündungszeichen völlig fehlen.

\section{Korrespondenzadresse}

\section{Dr. F. Wagner}

BG-Unfallklinik Murnau

Prof.-Küntscher-Straße 8, 82418 Murnau

fwagner@bgu-murnau.de

Interessenkonflikt. Der korrespondierende Autor gibt an, dass kein Interessenkonflikt besteht.

\section{Literatur}

1. Hagemann O (Hrsg) Laborlexikon: www.laborlexikon.de
2. Hendrich C, Frommelt L, Eulert J (Hrsg) (2004) Septische Knochen- und Gelenkchirurgie. Springer, Heidelberg

3. Hofmann GO (Hrsg) (2004) Infektionen der Knochen und Gelenke. Urban \& Fischer, München

4. Lorrot M, Fitoussi F, Faye A, et al (2007) Laboratory studies in pediatric bone and joint infections. Arch Pediatr [Suppl 2] 14:86-90

5. Mutschler W, Haas N (1999) Praxis der Unfallchirurgie. Thieme, Stuttgart

6. Peters KM, Koberg K, Kehren H, Zilkens KW (1991) PMN Elastase as a marker in diagnosis and follow up of bone and joint infections. Unfallchirurg 94(7):376-379

7. Rüter A, Trenz O, Wagner M (Hrsg) (2004) Unfallchirurgie, 2. Aufl. Urban \& Fischer, München

8. Schmelz A, Kinzl L, Einsiedel T (2006) Osteitis. Chirurg 77:943-962

9. Schnettler R, Steinau H-U (2004) Septische Knochenchirurgie. Thieme, Stuttgart

10. Unkilla-Kallio L, Kallio MJ, Eskola J, Peitola H (1994) Serum C-reactive protein, erythrocyte sedimentation rate and white blood cell count in acute haematogenous osteomyelitis of children. Paediatrics 93(1):59-62

11. Unkilla-Kallio L, Kallio MJ, Peitola H (1994) The usefulness of $\mathrm{C}$-reactive protein levels in the identification of concurrent septic arthritis in children who have acute haematogenous osteomyelitis. A comparison with the usefulness of the erythrocyte sedimentation rate and the white blood cell count. J Bone Joint Surg Am 76(6):848-853

12. Wagner FD, Hofmann GO, Bär T et al (1999) Infected non union. Osteosynthese Int [Suppl 1] 7:52-55

13. Weber BG, Czech O (1973) Pseudarthrosen. Huber, Bern 